\title{
Assessment of turbulent beam ion redistribution in tokamaks through velocity space-dependent gyrokinetic analyses
}

\author{
M. Albergante, A. Fasoli, J.P. Graves, S. Brunner and W.A. Cooper \\ Centre de Recherches en Physique des Plasmas, Association EURATOM-Confédération Suisse, \\ 1015 Lausanne, Switzerland \\ E-mail: mattia.albergante@epfl.ch
}

Received 15 November 2011, accepted for publication 13 March 2012

Published 3 September 2012

Online at stacks.iop.org/NF/52/094016

\begin{abstract}
We present the interface between a gyrokinetic code and a guiding centre code dedicated to the study of fast ion turbulent transport. A set of velocity space-dependent (kinetic) transport quantities, representing the link between the two codes, is presented. The code suite is applied to DEMO and TCV plasmas. While negligible alpha particle transport is observed for both tokamaks, important beam ion redistribution is obtained for simulations of DEMO. Results for the TCV tokamak demonstrate that the influence of turbulent fields on fast ion transport strongly depends on the plasma scenario.
\end{abstract}

(Some figures may appear in colour only in the online journal)

\section{Introduction}

Fast ion transport in thermonuclear devices can be caused by a variety of phenomena [1]. Some of these, such as the interaction with Alfvén waves [2], can lead to important losses [3] which might damage the device. Others are less disruptive but can still undermine performance in future burning plasma experiments. The interaction of fast ions with small scale turbulence belongs to this second category.

Until recently, microturbulent fields were conventionally expected to drive negligible transport levels for alpha particles, a consequence of beneficial phase space averaging effects [4]. However, a series of experimental [5,6], theoretical [7] and numerical investigations [8-10] demonstrated that this conclusion might not hold for energetic ions created by neutral beam injection: in general, transport can be significant for particles characterized by 'intermediate' energies $\left(E / T_{\mathrm{e}} \simeq 10\right)$ and small Larmor radii.

While limited consequences are foreseen for ITER [11], beam ion transport can become important for larger temperature machines, i.e. nuclear fusion power plants. Furthermore, it is conjectured that if the plasma $\beta$ is large enough, turbulence might be of concern not only for beam ions, but also for alphas [12].

In this work we study the interaction between suprathermal particles and the microturbulent fields endemic in magnetic confinement plasmas. The analysis is carried out by developing the mathematical framework needed to define a set of velocity space-dependent (kinetic) transport quantities. This approach is particularly useful when applied to Eulerian gyrokinetic codes and it is presented in section 2, where we also demonstrate the importance of diffusion over convection for fast ions. A rule-of-thumb estimate of the fast ion redistribution strength is also given. In section 3, we present the main features of two codes, VENUS [13-15] and GENE [16,17], which are combined to predict the fast ion anomalous transport in experimental scenarios. Particular attention is given to the extraction of kinetic diffusivities from the gyrokinetic code GENE, which are then used in a stochastic radial diffusion module recently added to the single particle code VENUS. The results obtained with the numerical platform are presented section 4, where turbulent beam ion transport is evaluated for DEMO [18] and the TCV [19] tokamak. Conclusions are drawn in section 5 .

\section{Evaluating the influence of fast ion turbulent transport on plasma performance}

In the recent literature $[8,9,12]$, the fast ion transport was studied by focusing on the anomalous diffusivity generated by the presence of turbulent fields. In general, the fast 
ion diffusivity decays with increasing energy, although the exact behaviour and the underlying physical mechanisms are still disputed $[9,12]$. The influence of magnetic fluctuation effects at finite $\beta_{\mathrm{e}}$ is also unclear as it might enhance fast ion transport [12] but also provides a stabilization for ITG [20]. Several other elements must be considered when predicting the behaviour and redistribution of fast ions in the presence of microturbulence.

Fast ions might experience a strong collisionality, especially in high-density plasmas. Therefore, their suprathermal character would only survive for a short time before thermalization. The reduced exposure time to turbulent fields would therefore render the effect of anomalous transport negligible. On the other hand, in plasmas of low collisionalities, the deceleration of fast ions would take longer. These could then diffuse on longer time scales and even small diffusivities could have a detrimental effect on plasma performance. We can therefore try and summarize the potential impact of anomalous fast ion transport by defining an overall figure of merit [21]

$$
\Delta=\frac{\text { Anomalous spread }}{\text { Minor radius }} \simeq \frac{\sqrt{\left\langle D_{v}\right\rangle \tau_{\mathrm{sd}}}}{a} .
$$

Here, $\left\langle D_{v}\right\rangle$ is the averaged diffusivity experienced by a fast ion population during the time $\tau_{\mathrm{sd}}$ required to thermalize it. Analytical estimates for $\tau_{\mathrm{sd}}$ can easily be obtained [22]. First principle estimates of $\left\langle D_{v}\right\rangle$ can also be calculated

$$
\langle D\rangle=\frac{\int \mathrm{d} E \sqrt{E} f_{\mathrm{sd}}(E) D_{v}(E)}{\int \mathrm{d} E \sqrt{E} f_{\mathrm{sd}}(E)}
$$

where $f_{\text {sd }}$ is a slowing down distribution function and $D_{v}(E)$ can be obtained, e.g., from the procedure described in [23]. The guidance of (1) and (2) can therefore provide a simple guess as to whether anomalous transport can play a role in a particular experiment. It was previously mentioned, however, that the behaviour of $D_{v}$ depends on the plasma scenario and cannot be determined a priori. Furthermore, the importance of convection has been largely ignored so far. To obtain reliable, well-founded projections on the fast ion behaviour in experimental scenarios, gyrokinetic simulations are then needed. Guiding centre test particle codes are also required to estimate the combined effect of turbulence and collisions in a global plasma environment.

\subsection{Velocity space-dependent transport quantities}

Gyrokinetic codes are commonly employed in the analysis of the anomalous particle and heat transport in tokamak plasmas. These codes, often used for investigations concerning background species, can be exploited for fast ion studies as well. The radial turbulent particle flux of an arbitrary species, is calculated as

$$
\Gamma=\left\langle\int \mathrm{d} v \delta f \delta u_{r}\right\rangle,
$$

where $\delta f$ represents a perturbation of the equilibrium distribution function $f_{0},\langle\rangle$ denotes a flux surface average and $\delta u_{r}$ is the radial projection of the particle drift in the turbulent fields

$$
\begin{gathered}
\delta u_{r}=\left(\delta \boldsymbol{u}_{E \times B}+\delta \boldsymbol{u}_{A_{\|}}\right) \cdot \boldsymbol{e}_{r}, \\
\delta \boldsymbol{u}_{E \times B}=-\frac{\nabla \delta \Phi \times \boldsymbol{B}}{B^{2}}, \\
\delta \boldsymbol{u}_{A_{\|}}=-v_{\|} \frac{\nabla \delta A_{\|} \times \boldsymbol{B}}{B^{2}} .
\end{gathered}
$$

Making use of a gyrokinetic code to estimate (3) for energetic particles is disadvantageous. One would need to solve the fast ion motion together with the slow evolution, on much shorter scales, of the background turbulence. A more convenient approach is presented here, where two different codes are used to decouple the interaction with turbulence, from its consequences, i.e. the fast ion transport. Diffusion and convection can then be obtained by studying the background turbulent fields with gyrokinetic simulations. Then, guiding centre simulations with ad hoc Monte Carlo operators can provide estimates of the actual transport levels of the energetic ions in the presence of other physical phenomena.

Some limitations, frequently found in gyrokinetic codes, must be overcome. A $\delta f$ scheme is used to obtain (3), therefore assuming an equilibrium distribution $f_{0}$ that is difficult to assess for fast ions. Fast ions collide with the background plasma and their velocity significantly changes from birth to full thermalization. As we will see, in the formulations used here, the gyrokinetic code is employed to obtain velocity spacedependent (kinetic) transport quantities for single particles, i.e. without the need to define a specific $f_{0}$. Kinetic diffusion and convection are then used in the guiding centre code, where a full- $f$ scheme is applied.

Definition of integrated transport quantities. Let us first consider $\delta f$, calculated from the gyrokinetic equation

$$
\begin{aligned}
\frac{\partial \delta f}{\partial t} & +\left(\boldsymbol{v}_{\mathrm{gc}}+\delta \overline{\boldsymbol{u}}\right) \cdot \nabla \delta f+\delta \overline{\boldsymbol{u}} \mu \nabla B \frac{\partial \delta f}{\partial E} \\
& =-\delta \overline{\boldsymbol{u}} \boldsymbol{\nabla} f_{0}+\left(\boldsymbol{v}_{\mathrm{gc}}+\delta \overline{\boldsymbol{u}}\right) Q\left(\boldsymbol{\nabla} \delta \bar{\Phi}+\boldsymbol{b}_{0} \delta \bar{A}_{\|}\right) \frac{\partial f_{0}}{\partial E}
\end{aligned}
$$

In our conventions, an overbar indicates a gyroaveraged quantity. $\boldsymbol{v}_{\mathrm{gc}}$ is the motion of a particle with charge $Q$ in the presence of the equilibrium magnetic field only, $\boldsymbol{b}_{0}$ is the unit vector along $\boldsymbol{B}_{0}$ and $\boldsymbol{\nabla}$ the configuration space gradient. The quantity $\delta \overline{\boldsymbol{u}}$ is calculated with the definitions of equation (4) and the employment of the gyroaveraged perturbed fields $\delta \bar{\Phi}$ and $\delta \bar{A}_{\|}$. Equation (7) can be written in symbolic notation, similarly to [24] and [25], to ease the mathematical treatment

$\hat{\boldsymbol{G}} \delta f=(\hat{\boldsymbol{L}}+\delta \hat{\boldsymbol{L}}) \delta f=-\left(\delta \overline{\boldsymbol{u}} \cdot \nabla f_{0}+F \frac{\partial f_{0}}{\partial E}\right)$,

$\hat{\boldsymbol{L}}=\frac{\partial}{\partial t}+\boldsymbol{v}_{\mathrm{gc}} \cdot \nabla$

$\delta \hat{\boldsymbol{L}}=\delta \overline{\boldsymbol{u}} \cdot \nabla+\delta \overline{\boldsymbol{u}} \cdot \mu \nabla B \frac{\partial}{\partial E}$.

$\hat{G}$ is the gyrokinetic operator and

$$
F \equiv-\left(\boldsymbol{v}_{\mathrm{gc}}+\delta \bar{u}\right) Q\left(\nabla \delta \bar{\Phi}+\delta \bar{A}_{\|}\right)
$$

Equation (7) can be solved for $\delta f$ by inverting the gyrokinetic operator

$\delta f=-\hat{\boldsymbol{G}}^{-1}\left\{\nabla f_{0} \cdot \delta \overline{\boldsymbol{u}}\right\}-\hat{\boldsymbol{G}}^{-1}\left\{F \partial_{E} f_{0}\right\}=\delta f_{1}+\delta f_{2}$. 
$\hat{G}^{-1}$ represents an integration along the perturbed particle motion. The particle flux (3) can thus be split into two components

$$
\int \mathrm{d} \boldsymbol{v} \delta f \delta \boldsymbol{u}=\underbrace{\int \mathrm{d} \boldsymbol{v} \delta f_{1} \delta \boldsymbol{u}}_{\Gamma_{1}, \text { (thermo)diffusive }}+\underbrace{\int \mathrm{d} \boldsymbol{v} \delta f_{2} \delta \boldsymbol{u}}_{\Gamma_{2}, \text { convective }} .
$$

Note that the nomenclature '(thermo)diffusive' and 'convective' is purely conventional. It separates the fluxes driven by $\nabla f_{0}$ by those who do not depend on density gradients of the distribution. We now extend the definition of these quantities from an integrated to a velocity space dependent, kinetic, framework.

Definition of kinetic transport quantities. We dedicate this section to the introduction of a series of kinetic transport quantities that can be used in gyrokinetic codes to estimate fast ion transport. Let us focus the analysis on passive tracers, i.e. particles that are of negligible concentrations in the plasma. This approximation is accurate for highly diluted energetic ions, as recently demonstrated with gyrokinetic simulations $[25,26]$. The mathematical framework describing fast ions is considerably simplified in the tracer approximation.

Let us recall the velocity space-dependent particle diffusivity introduced recently in [10]

$$
D_{v}=-\frac{\delta f_{1} \delta u_{r}}{\nabla f_{0}}
$$

With the formalism employed in the previous section, this corresponds to

$$
D_{v}=\frac{\hat{\boldsymbol{G}}^{-1}\left\{\boldsymbol{\nabla} f_{0} \cdot \delta \overline{\boldsymbol{u}}\right\} \delta u_{r}}{\nabla f_{0}}=\frac{\hat{\boldsymbol{G}}^{-1}\left\{\nabla_{r} f_{0} \delta \bar{u}_{r}\right\} \delta u_{r}}{\nabla_{r} f_{0}} .
$$

Here, we have assumed gradients in $f_{0}$ to be present only in the radial direction. 'Electrostatic' and 'magnetic' diffusivities commonly discussed in the literature can be obtained from (15)

$$
\begin{gathered}
D_{v}^{\mathrm{es}}=\frac{\hat{\boldsymbol{G}}^{-1}\left\{\nabla f_{0} \delta \bar{u}_{r}\right\} \delta u_{E \times B}}{\nabla f_{0}}, \\
D_{v}^{\mathrm{m}}=\frac{\hat{\boldsymbol{G}}^{-1}\left\{\nabla f_{0} \delta \bar{u}_{r}\right\} \delta u_{A_{\|}}}{\nabla f_{0}} .
\end{gathered}
$$

Velocity space convection can be defined similarly to (15) as

$$
C_{v}=\frac{\hat{G}^{-1}\left\{F \partial_{E} f_{0}\right\} \delta u_{r}}{\partial_{E} f_{0}} .
$$

The kinetic transport quantities $D_{v}$ and $C_{v}$ still depend on $f_{0}$. This choice would be undesirable, given that in the passive approach the turbulent fields and the transport they generate do not vary with $f_{0}$. We now demonstrate that such dependence is only apparent.

Validation of kinetic diffusion. Let us consider the definition of a kinetic diffusivity

$$
D_{v}=\frac{\hat{G}^{-1}\left\{\nabla_{r} f_{0} \delta \bar{u}_{r}\right\} \delta u_{r}}{\nabla f_{0}}=D_{v}^{L}+\delta D_{v} .
$$

Here, $D_{v}^{L}$ represents the 'unperturbed' part of $D_{v}$

$$
D_{v}^{L}=\frac{\left[\nabla_{r} f_{0} \hat{\boldsymbol{L}}^{-1}\left(\delta \bar{u}_{r}\right)\right]}{\nabla_{r} f_{0}} \delta u_{r}=\left[\hat{\boldsymbol{L}}^{-1}\left(\delta \bar{u}_{r}\right)\right] \delta u_{r},
$$

Being $\hat{\boldsymbol{L}} f_{0}=0$ and $\nabla_{r} f_{0} / f_{0}=$ const. in the local approximation, we could extract $\nabla_{r} f_{0}$ from the operator $\hat{\boldsymbol{L}}^{-1}$ in (20). The quantity $D_{v}^{L}$ in (20) is therefore independent of $\nabla_{r} f_{0}$. As a consequence, $\delta D_{v}$ includes the difference between the 'unperturbed' treatment and the employment of the full 'perturbed' operator. If this component were important, $D_{v}$ would vary with $\nabla f_{0}$ and our definition of kinetic transport quantities would be questionable. Let us perform a numerical simulation with the GENE code $[16,17]$ to quantify the importance of $\delta D_{v}$. We use the local version of the code and simulate a plasma discharge of the TCV tokamak [19], which was investigated in a recent publication [27]. We use the same parameters as in [27] but, in this part of the analysis, we assume an $s-\alpha$, geometry. This choice allows other research groups to run simple simulations for benchmarking purposes. After simulating the background turbulence, we introduce in the Eulerian simulation several passive species characterized by different density and temperature gradients, i.e. different $\nabla_{r} f_{0}$. In figure 1 we clearly observe similar values of $D_{v}$ for passive populations that are characterized by different $\Omega_{n}$ and $\Omega_{T}$

$$
\begin{aligned}
& \Omega_{T}=\frac{R_{0}}{a} \frac{1}{T} \frac{\partial T}{\partial \rho_{t}}, \\
& \Omega_{n}=\frac{R_{0}}{a} \frac{1}{n} \frac{\partial n}{\partial \rho_{t}} .
\end{aligned}
$$

The values of $\Omega_{T}$ and $\Omega_{n}$ in figure 1 are only varied for the tracer fast ion population as the background plasma profiles, and thus turbulence properties, are kept fixed. Only minor differences in $D_{v}$ for the three populations are observed in the low energy region. This discrepancy is due to the normalization with respect to a Maxwellian characterized only by temperature gradients. In such a case, the quantity $D_{v}$ is obtained as follows

$$
D_{v}=-\frac{\delta f \delta u_{r}}{\nabla_{r} f_{0}} \propto \frac{1}{E / T_{\text {passive }}-3 / 2} .
$$

The vanishing quantity at the denominator is responsible for the disagreement observed in the low-energy region of figure 1 .

We can conclude that, since $D_{v}$ is virtually independent of $\nabla f_{0}, \delta D_{v}$ is small and therefore $\hat{G} \simeq \hat{\boldsymbol{L}}$, at least for the case studied here. With this result we have therefore proven that, for realistic conditions, kinetic diffusion (e.g. equation (15)) is independent of $f_{0}$. Kinetic transport coefficients can be obtained from gyrokinetic simulations with the employment of an arbitrary $f_{0}$. A drift kinetic code can then be used to solve the particle motion in its entirety, i.e. including particle sources and sinks, collisions and many other physical phenomena. We assume that similar results hold for $C_{v}$ defined in (18). The Maxwellian $f_{0}$ assumed in the GENE code prevents us from performing more detailed investigations but, as we shall see in the next section, fast ion convection is marginal. 

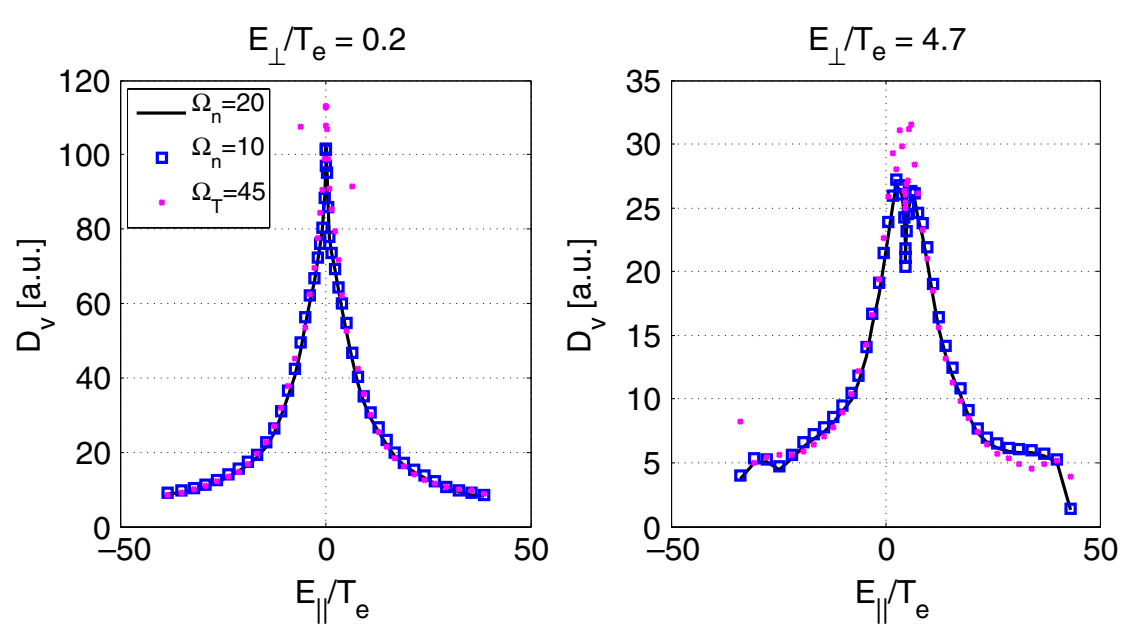

Figure 1. Comparison between the velocity space-dependent diffusivity of three fast ion populations characterized by different density and temperature gradients and embedded in the same ITG/TEM turbulence. When $\Omega_{T}$ is indicated, $\Omega_{n}$ is equal to zero and viceversa.
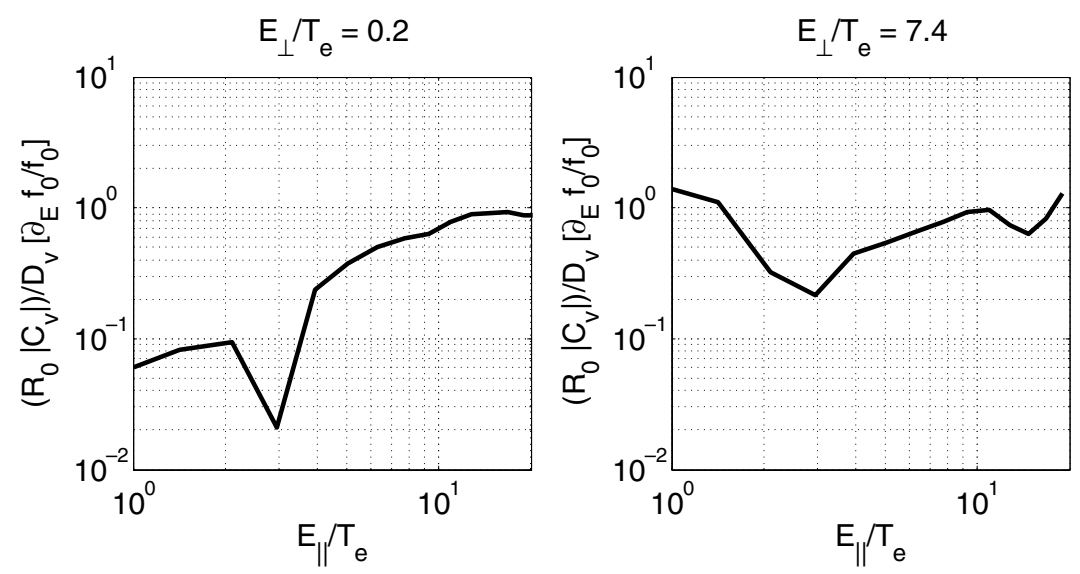

Figure 2. Particle convection to diffusion ratio as a function of the passive ion parallel energy. Convection, whose absolute value is considered here, is a minor phenomenon over a wide range of energies.

Prevalence of diffusion over convection for fast ion transport. In a work by Zhang and co-workers it was observed that fast ion transport in tokamaks is diffusive [9]. Similar results are obtained in our simulations for the relevant case where ions have a peaked density profile (large $\nabla \ln f_{0}$ ). In this case, we can estimate the relative influence of diffusion and convection on the particle flux

$$
\begin{aligned}
\frac{\Gamma_{v}}{f_{0}} & =-D_{v} \nabla_{r} \ln f_{0}+C_{v} \frac{\partial_{E} f_{0}}{f_{0}} \\
& =-D_{v} \nabla_{r} \ln f_{0}\left[1-\frac{R_{0} C_{v}}{D_{v}} \frac{\partial_{E} f_{0}}{f_{0}} \frac{1}{R_{0} \nabla_{r} \ln f_{0}}\right] .
\end{aligned}
$$

Although the quantity $\frac{R_{0} C_{v}}{D_{v}} \frac{\partial_{E} f_{0}}{f_{0}}$ strongly varies with the particle energy, it is generally below unity (figure 2). Furthermore, energetic ions from fusion burn and neutral beam injection are characterized by strongly peaked distribution functions, for which $R_{0} \nabla \ln f_{0} \gg 1$. We consequently observe dominant diffusion in the energetic particle motion in turbulent fields

$$
\begin{aligned}
\frac{\Gamma_{v}}{f_{0}} & =-D_{v} \nabla_{r} \ln f_{0}[1-\underbrace{\frac{R_{0} C_{v}}{D_{v}} \frac{\partial_{E} f_{0}}{f_{0}}}_{<1} \underbrace{\frac{1}{R_{0} \nabla_{r} \ln f_{0}}}_{\ll 1}] \\
& \simeq-D \nabla_{r} \ln f_{0} .
\end{aligned}
$$

In some scenarios, however, a resonant interaction between fast particles and electromagnetic waves (i.e. Alfvén eigenmodes) could lead to the flattening of the fast ion density profile [28]. Fast ion populations characterized by flat density profiles, i.e. $R_{0} \nabla \ln f_{0} \simeq 1$, could therefore be subject to convective phenomena, as suggested by equation (25) and the results in figure 2. The nonlinear interaction between Alfvén eigenmodes, collisions and turbulence would therefore require more detailed studies, such as those presented in [29].

For the rest of this work we concentrate on particle diffusion. A series of publications already focused on this point, concluding that particle diffusivity decreases with particle energy $[9,12]$, due to gyroaveraging effects (see, e.g., equation (20) with the definitions of (4)). So far, however, the consequences for plasma operations were only assessed in terms of the quantity $D_{v}$. As we shall see shortly, the fast ion collisionality also plays an important role.

\section{The updated version of the VENUS code}

The updated version of the VENUS code [13-15] and its interface with GENE are used to assess the anomalous fast ion redistribution. The numerical platform [11] simulates the 


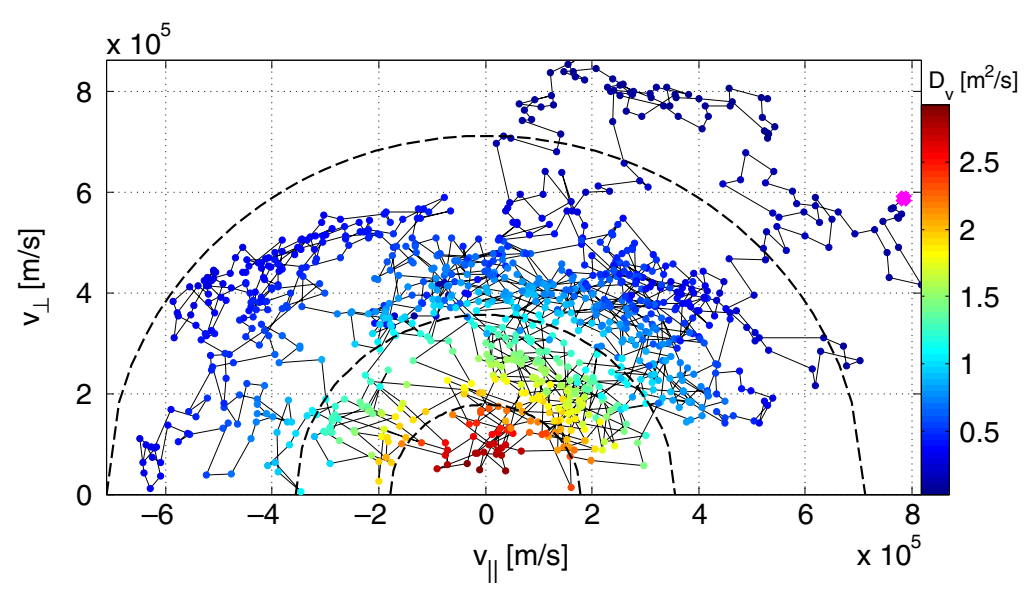

Figure 3. Slowing down of a neutral beam particle (birth position is indicated in magenta) in the TCV tokamak. The $25 \mathrm{keV}$ deuterium particle transfers its energy until background thermal velocities are obtained (dashed lines represent 1,2 and $4 v_{\text {th,i }}$ ). The colour code represents the corresponding kinetic diffusion coefficient experienced by the particle during the thermalization process, in $\mathrm{m}^{2} \mathrm{~s}^{-1}$.

particle trajectory in the presence of equilibrium magnetic fields, Coulomb collisions and anomalous transport. The platform is tailored to describe the behaviour of beam ions and for this purpose a beam deposition module was added to VENUS. Given plasma parameters and the geometry of the neutral beam injection system, the beam source for the VENUS code can be obtained with the procedure described in [11].

The Coulomb collision operator. The Coulomb collision operator was recently upgraded in the VENUS code [30-32]. At each time step, the particle $i$ experiences stochastic kicks in energy and pitch angle

$$
\begin{aligned}
\Delta E_{i}= & -\sum_{j} 2\left(v_{E_{i j}} \Delta t\right)\left[E_{i}-\left(\frac{3}{2}+\frac{E_{i}}{v_{E_{i j}}} \frac{\mathrm{d} v_{E_{i j}}}{\mathrm{~d} E_{i}}\right) T_{j}\right] \\
& +2 \mathcal{R} \sqrt{\sum\left(T_{j} v_{E_{i j}}\right) E_{i} \Delta t}, \\
\Delta \lambda_{i}= & -\sum_{j} \lambda_{i} v_{\lambda_{i j}} \Delta t+\mathcal{R} \sqrt{\left(1-\lambda_{i}^{2}\right) \sum v_{\lambda_{i j}} \Delta t},
\end{aligned}
$$

where $\lambda=v_{\|} / v, \Delta t$ is the step size, $\mathcal{R}$ can either be \pm 1 with equal probability and the sum is performed over the background species (labelled with $j$ ). Collision frequencies between the fast particle $i$ and the background species $j$ are

$$
\begin{gathered}
v_{\lambda_{i j}}=\left[\frac{Q_{i}^{2} Q_{j}^{2} \ln \Lambda_{j} n_{j}}{4 m_{i}^{2} \pi \varepsilon_{0}^{2}}\right] \frac{\phi\left(x_{i j}\right)-\Psi\left(x_{i j}\right)}{v_{i}^{3}}, \\
v_{E_{i j}}=\left[\frac{Q_{i}^{2} Q_{j}^{2} \ln \Lambda_{j} n_{j}}{4 m_{i}^{2} \pi \varepsilon_{0}^{2}}\right] \frac{m_{i}}{m_{j}} \frac{\Psi\left(x_{i j}\right)}{v_{t h j}^{2} v_{i}},
\end{gathered}
$$

where $\ln \Lambda$ is the Coulomb logarithm [22] and

$$
\begin{gathered}
x_{i j}=v_{i} / v_{t h, j}, \\
v_{t h, j}=\sqrt{\frac{2 T_{j}}{m_{j}}}, \\
\phi(z)=\operatorname{erf}(z)=\frac{2}{\sqrt{\pi}} \int_{0}^{z} \mathrm{e}^{-y^{2}} \mathrm{~d} y, \\
\Psi(z)=\frac{\phi(z)-z \phi^{\prime}(z)}{2 z^{2}} .
\end{gathered}
$$

The number of background species is now completely arbitrary. This part of the numerical platform was benchmarked in a recent work where the code successfully reproduced the fast ion distribution function probed with the CTS diagnostic in TEXTOR [33].

Description of turbulent transport in VENUS. Anomalous transport is modelled with a stochastic radial diffusion term based on the transport coefficients simulated by GENE. Fast particles experience a radial kick [34]

$\Delta s(s, \boldsymbol{v})=\mathcal{R} \sqrt{2\left(\frac{4 D_{v}(\boldsymbol{v}) s}{a^{2}}\right) \Delta t}+\left(\frac{4 D_{v}(\boldsymbol{v})}{a^{2}}\right) \Delta t$,

with $s$ the normalized toroidal flux, $\mathcal{R}$ a normally distributed random number, $a$ the tokamak minor radius and $\Delta t$ the time step. The velocity-dependent radial diffusion $D_{v}$ is obtained from GENE as discussed in the previous section for arbitrary $f_{0}$. It is used by VENUS to evaluate equation (34) at each time-step during the thermalization of the particle, a process which moves the particle to lower energy (i.e. large diffusivity) regions. An example is visible in figure 3, where the different diffusivities experienced by particles during their slowing down are represented on a colour scale.

Extension to a global-like version of the code. One limitation of our approach is the employment of the local version of the GENE code to obtain the diffusion coefficient $D_{v}$. To overcome this limitation and to obtain an estimate for $D_{v}$ for different radial positions, two techniques are used in this work. For the simulations of TCV, several flux-tube simulations are performed at different radial locations. A normalization is then imposed to ensure that the diffusivity is consistent with what is observed for the background plasma. In other words, we verify that $D_{v}^{\rho_{t}}$ satisfy the relation

$$
D_{\mathrm{GENE}}\left(\rho_{t}\right) \equiv \frac{\int \mathrm{d} \boldsymbol{v} D_{v}^{\rho_{t}}(\boldsymbol{v}) f_{0}(\boldsymbol{v})}{\int \mathrm{d} \boldsymbol{v} f_{0}(\boldsymbol{v})}=D_{\mathrm{xpt}}\left(\rho_{t}\right),
$$

where $D_{v}^{\rho_{t}}$ is obtained from a GENE simulation at $\rho_{t}, f_{0}$ is a Maxwellian with a background temperature and $D_{\text {xpt }}$ is the 

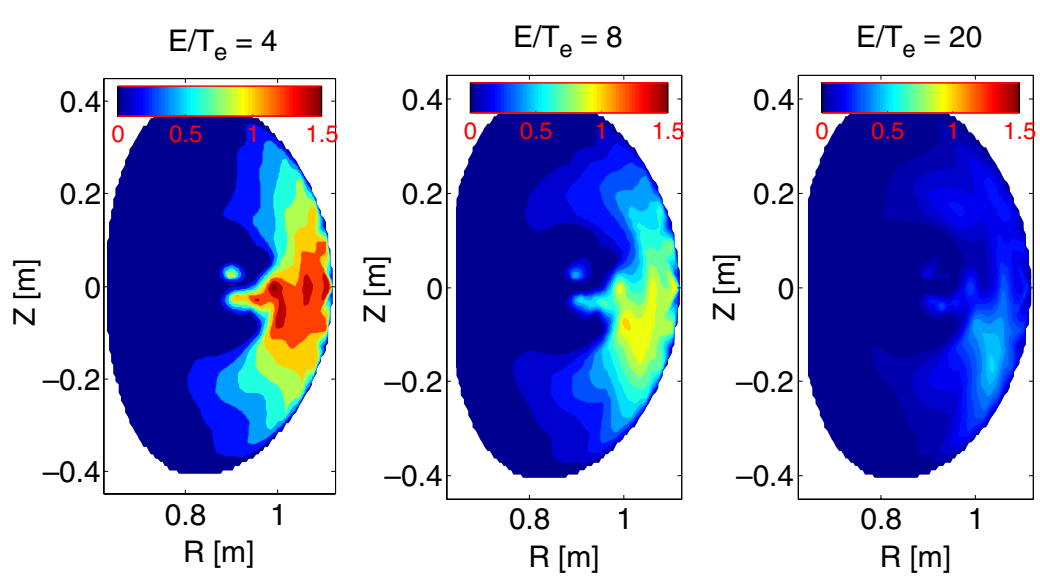

Figure 4. Particle diffusivity $D_{v}$ of a strongly passing beam ion $(\lambda \simeq 1)$ as a function of the particle position in the TCV tokamak. The results, obtained with the GENE code, are shown for three different energies to appreciate the transport suppression.

experimental diffusivity of $f_{0}$. In figure 4 we observe the fast ion diffusivity of strongly passing particles at different positions in the plasma. For the analysis of DEMO, this approach is unpractical given the large number of grid points required for each flux-tube simulation. The particle diffusivity is then obtained at mid-radius and assumed to be radially constant

$$
D_{v}\left(\rho_{t}, \boldsymbol{v}\right)=D_{v}\left(\rho_{t}=0.5, \boldsymbol{v}\right) .
$$

It was shown that this choice gives results in qualitative agreement with (35) for the TCV tokamak [34]. We therefore employ this convenient model for DEMO to save computational power.

\section{DEMO and TCV simulations}

The results presented shortly are an extension of the observations of [11], where it was concluded that the turbulent transport of beam ions and alpha particles is negligible in ITER. While the observation of a negligible transport for alphas is found in all the machines tested $\left(E_{\alpha} / T_{\mathrm{e}} \gtrsim 140\right)$, the same cannot be said about neutral beam ions. In co-injecting neutral beam systems these particles are characterized by small gyroradii, and also negligible gyroaveraging effects. We now show that the large temperatures envisaged in reactors such as DEMO results in a smaller $E_{\mathrm{nbi}} / T_{\mathrm{e}}$ and in long times to thermalize the particles. Similar conclusions will be drawn for TCV, where the small $E_{\mathrm{nbi}} / T_{\mathrm{e}}$ ratio is mostly responsible for anomalous redistribution. A survey of the parameters characterizing the numerical simulations of DEMO and TCV plasmas can be found on table 1 .

\subsection{Fast ion transport in DEMO}

To define a reference scenario for DEMO, we take inspiration from recent work where the key features of this future fusion reactor were outlined [18]. It was concluded that DEMO will be larger than ITER, with a major radius of $8.5 \mathrm{~m}$. A large magnetic field is also envisaged, chosen in our simulations to reach $6 \mathrm{~T}$. Particle densities are expected to be constrained by the Greenwald limit and will therefore resemble those of ITER. Large temperatures are then needed to obtain a large fusion yield. For the analysis carried out in this work, we assume
Table 1. Main features of the plasma discharges investigated in the TCV tokamak and in DEMO.

\begin{tabular}{llll}
\hline & TCV, \#27489 & TCV, \#25013 & DEMO \\
\hline$n_{\mathrm{e} 0}\left(\times 10^{19} \mathrm{~m}^{-3}\right)$ & 4.5 & 1.9 & 8 \\
$T_{\mathrm{e} 0}(\mathrm{keV})$ & $<1$ & 12 & 50 \\
$q_{\text {min }}$ & 2.5 & 2 & $\simeq 2$ \\
Beam energy & $25 \mathrm{keV}$ & $25 \mathrm{keV}$ & $1.5 \mathrm{MeV}$ \\
$\begin{array}{l}\text { Numerical } \\
\text { particles }\left(\times 10^{5}\right)\end{array}$ & 3 & 3 & 1.2 \\
Simulated time $(\mathrm{s})$ & 0.2 & 0.1 & 6 \\
Injection times & 250 & 300 & 150 \\
\hline
\end{tabular}

the background profiles to be the same as those envisaged for the ITER steady-state scenario [35]. The core density is increased from $n_{\mathrm{e} 0}^{\text {iter }}=6 \times 10^{19} \mathrm{~m}^{-3}$ to $n_{\mathrm{e} 0}^{\text {demo }}=8 \times 10^{19} \mathrm{~m}^{-3}$. The peak temperature is increased by a larger factor, from $T_{\mathrm{i} 0}^{\mathrm{iter}} \simeq 30 \mathrm{keV}$ to $T_{\mathrm{i} 0}^{\text {demo }} \simeq 55 \mathrm{keV}$. We model the NBI system as a single, co-injecting PINI providing energetic beam ions at $1.5 \mathrm{MeV}$ (full energy). Half of the beam power, here assumed to be approximately $70 \mathrm{MW}$, is equally divided between the half- and third-energy populations. The large temperatures and the $\mathrm{MeV}$-injection are responsible for a long slowing down time. The beam ion population is fully thermalized after approximately four seconds, much more than the similar NBI system in ITER. Anomalous redistribution is then foreseen, as suggested by (1) with which we obtain $\Delta_{\text {DEMO }} \simeq 0$.8. For ITER, we similarly obtained $\Delta_{\text {ITER }} \simeq 0.3$ and virtually no redistribution [11]. Numerical simulations with the VENUS code are performed to obtain more comprehensive results.

The turbulent fields responsible for the background particle and heat transport in DEMO are, as of today, unknown. As a consequence, there is uncertainty regarding the fast ion behaviour in DEMO's microturbulent fields. We choose to employ the fast ion diffusivities obtained in the recent work where ITER's neutral beam particle transport was studied [11]. It was shown that the microturbulence underlying the particle transport was generated by a mixture of ITG and TEM microinstabilities. Finite $\beta$ effects were retained in the analysis, contributed to the partial stabilization of modes drifting in the ion acoustic direction and carried negligible transport of energetic ions. The fast ion diffusivity used in the analysis of DEMO is chosen to be identical to that 


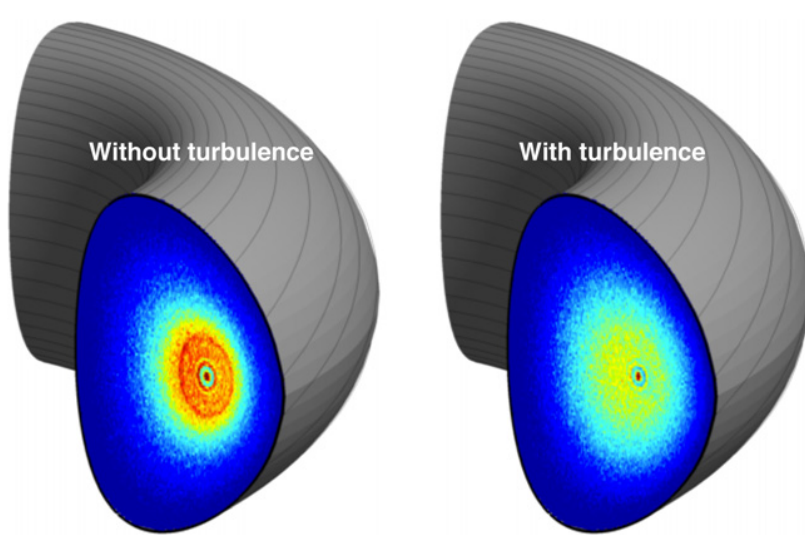

Figure 5. Beam ion density in DEMO. The left panel describes the results obtained with only the inclusion of collisional transport. The results in the right panel are obtained by also including turbulent transport.

used in recent ITER simulations [11]. The shape of the function $D_{v}\left(\rho_{t}, \theta, E_{\mathrm{nbi}} / T_{\mathrm{e}}, \lambda=v_{\|} / v\right)$ is also assumed to remain unchanged, while a renormalization is applied to take into account a large gyro-Bohm diffusivity in DEMO, given the larger temperatures achieved

$$
D_{v}^{\mathrm{demo}}\left(\rho_{t}, \theta, E / T_{\mathrm{e}}, \lambda\right)=A \cdot D_{v}^{\mathrm{iter}}\left(\rho_{t}, \theta, E / T_{\mathrm{e}}, \lambda\right) .
$$

The ratio between the gyro-Bohm diffusivity of the two scenarios provides the constant $A$

$$
A=\frac{D_{\mathrm{gb}}^{\text {demo }}}{D_{\mathrm{gb}}^{\text {iter }}}=\left[\frac{T_{\mathrm{e}, \text { demo }}}{T_{\mathrm{e}, \text { iter }}}\right]^{3 / 2} \frac{B_{\text {iter }}}{B_{\text {demo }}} \simeq 2.5 .
$$

We base our analysis on the assumption that the same turbulence will characterize ITER and DEMO operation. Still, a strong uncertainty remains on the features of the microturbulent fields in future plasmas at large $\beta$ [36].

The results in figure 5 highlight that ion redistribution of the $1.5 \mathrm{MeV}$ neutral beam ions is possible in DEMO. In the left panel of the figure we observe the two-dimensional distribution of energetic particles in DEMO in the absence of turbulent diffusion. A clear displacement of particles from the core to peripheral plasma regions is observed when the radial diffusion coefficient from GENE is included in the simulation (right panel). Energetic particles therefore thermalize in colder plasma regions where the critical energy $E_{\mathrm{c}}$ is lower. Beam ions consequently give more energy than expected to electrons rather than ions (figure 6). The importance of ion heating in DEMO is of primary importance for the machine [18] and the effect observed in figure 6 would be strongly undesirable. Furthermore, stronger redistribution was observed for lower density plasmas and for an alternative neutral beam system at $1 \mathrm{MeV}$ [34]. For this reason, present day experiments must contribute to the knowledge of this interaction, and shed light on how to avoid and control it.

\subsection{Anomalous redistribution of the planned NBI upgrade of TCV}

Several tokamaks and basic plasma experiments are performing experiments to examine the interaction between fast ions and turbulence. A future candidate for such analyses is the Tokamak à Configuration Variable (TCV). A number of plasma scenarios can be achieved thanks to the powerful and flexible ECH system delivering up to 4.5 MW of electron heating. Together with the flexibility on plasma shaping and density, it would represent an ideal experiment for studying the turbulent fast ion transport once the planned $25 \mathrm{keV}$ neutral beam heating upgrade has been installed [37].

The particle diffusivity employed in the VENUS analysis is obtained with GENE simulations of TCV plasma discharge \#29866 and presented in [27]. The reversed-shear scenario studied in [27] by Lapillonne is particularly suitable for the analysis as it is characterized, similarly to ITER simulations, by ITG and TEM turbulence. In figure 7 we can observe the resulting fast ion diffusivity at $\theta=0$ (i.e. the outboard mid-plane). The birth points of the beam ions in different plasma discharges are also represented. An important remark is that for $E / T_{\mathrm{e}}=100$, corresponding to alpha particles in ITER, fast ion diffusivities are below measurable levels. Alphas are therefore unaffected by turbulence, thus corroborating previous results obtained with different parameters [11].

The outcome of two numerical simulations is now presented, one where no redistribution is observed (plasma discharge \#27489) and one with strong redistribution (\#25013). In the first case, the background plasma is characterized by large plasma density $n_{\text {plasma, } 0}=4.5 \times 10^{19} \mathrm{~m}^{-3}$ and electron temperature below $1 \mathrm{keV}$. Consequently, a large $E_{\mathrm{nbi}} / T_{\mathrm{e}} \gtrsim 25$ ratio and a small fast ion slowing down time $\tau_{\mathrm{sd}}<10^{-2} \mathrm{~s}$ are envisaged, which result in a small redistribution $\left(\Delta_{27489}^{\mathrm{TCV}}<\right.$ 0.1 ). On the other hand, plasma discharge \#25013 is a hightemperature $\left(T_{\mathrm{e}} \simeq 12 \mathrm{keV}\right)$, intermediate-density $\left(n_{\text {plasma }, 0} \simeq\right.$ $2 \times 10^{19} \mathrm{~m}^{-3}$ ) ITB scenario. Long slowing down times $\left(\tau_{\mathrm{sd}} \simeq 10^{-1} \mathrm{~s}\right)$ and small $E_{\mathrm{nbi}} / T_{\mathrm{e}} \simeq 2$ could result in strong redistribution $\left(\Delta_{25013}^{\mathrm{TCV}}=0.75\right)$. Results are confirmed by VENUS simulations employing $N=3 \times 10^{5}$ deuterium particles (figure 8). In the left panel of the figure we observe that turbulence strongly changes the fast ion power deposition profile for plasma discharge \#25013. Particles are therefore displaced towards peripheral plasma regions where they heat the ions less effectively, an effect similar to what observed for DEMO. Although particle losses were not enhanced by turbulence in DEMO, this is the case for the TCV tokamak. Of the power reaching the plasma boundary, more than half is lost due to turbulent fields. Results are more optimistic for plasma discharge \#27489, for which efficient heat deposition is observed. The power given to the background ions is unaltered between simulations with and without the inclusion of a radial diffusion coefficient. We emphasize that results for plasma discharge \#25013 are scaled up by a factor 50 in figure 8 . Given the larger temperature of the background, beam ions transfer less energy to the plasma and can be considered as moderately suprathermal particles.

Overall, our results suggest that fast ion turbulent plasma in the TCV tokamak can either be very strong or negligible. By changing the plasma temperature and density, several scenarios with intermediate redistribution can be obtained. The results for such cases, i.e. all plasma discharges between \#25013 and \#27489 in figure 7, can be found in [34]. An equally important 

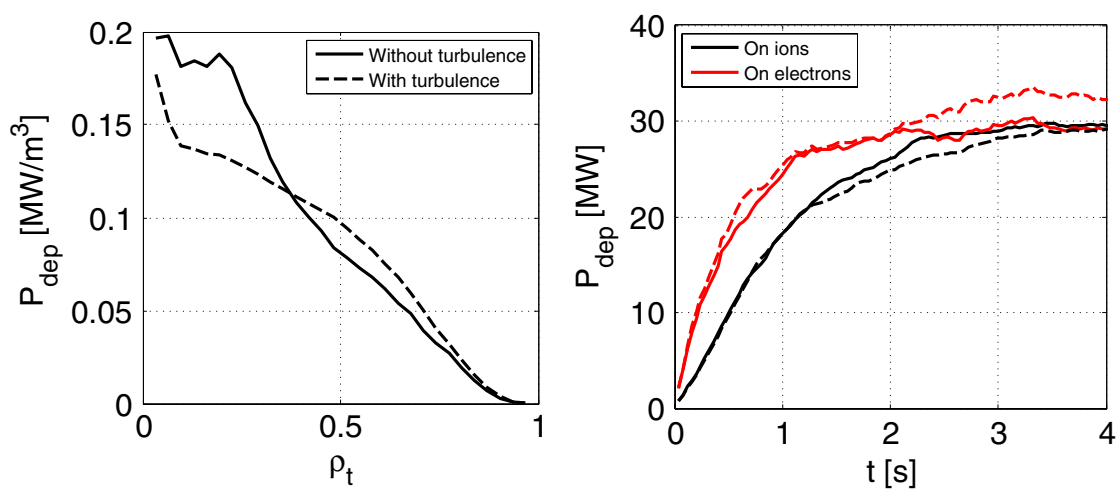

Figure 6. Heat deposition from the neutral beam system in DEMO. In the left panel the deposition profile with and without anomalous transport (dashed and solid lines, respectively) is illustrated. The power given to ions (black) and electrons (red) is also illustrated in the right panel.

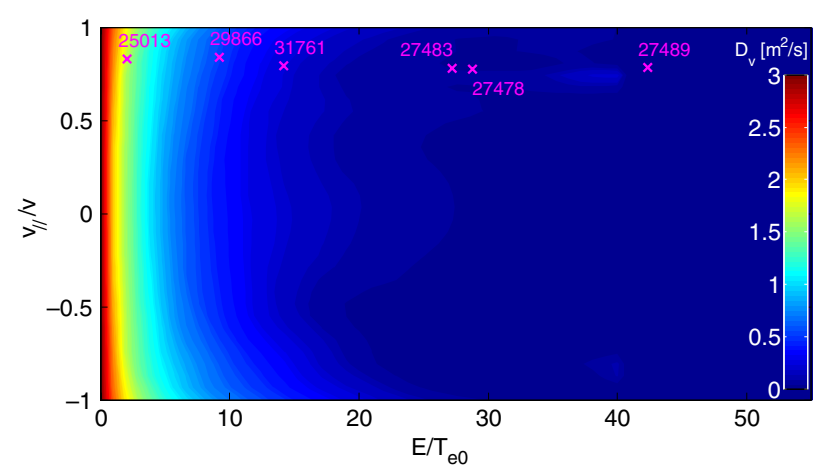

Figure 7. Injection energy, normalized to the plasma temperature, for some characteristic TCV plasma discharges. The particle diffusivity expected at mid-radius, $\theta=0$, for plasma discharge \#29866 is also represented.

feature of the TCV tokamak is the possibility of investigating plasmas with different shape and negative triangularity. Such topologies are characterized by weaker turbulent fields [38] and could be used for insightful studies on the optimal plasma shape to suppress fast ion transport in burning plasma devices.

\subsection{Comment on magnetic transport}

In a recent publication [12] it was shown that turbulent magnetic fluctuations can generate strong radial drifts, especially for passing particles. It was shown with elemental analytical calculations that magnetic diffusivity remains constant for particles with large $\lambda=v_{\|} / v$, irrespective of their energy. Given that electrostatic diffusivity decays with the particle energy, there is a point where $D_{v}^{\mathrm{m}}>D_{v}^{\mathrm{es}}$. Since $D_{v}^{\mathrm{m}}$ is proportional to $\beta_{\mathrm{e}}$, it was concluded that beam ions and alpha particles would be lost in burning plasmas. In the low $\beta$ analysis of TCV the intensity of magnetic perturbations was too low to carry an effect. Magnetic transport was negligible also in DEMO simulations where plasma pressure was large, $\beta_{\mathrm{e}}=1.5 \%$. Further investigations revealed that the magnetic diffusivity $D_{v}^{\mathrm{m}}$ remains fairly constant for large particle energies and $\lambda \simeq 1$, but nevertheless negligible. The crossing point, i.e. the energy $E_{\mathrm{cp}}$ at which

$$
D_{v}^{\mathrm{es}}\left(E_{\mathrm{cp}}\right)=D_{v}^{\mathrm{m}}\left(E_{\mathrm{cp}}\right)
$$

is found at very large energies, $E_{\mathrm{cp}} / T_{\mathrm{e}}>50$. Diffusivities are so low in this energy range that they are irrelevant for transport studies, even if $D_{v}^{\mathrm{m}}$ remains constant with increasing energy. It should also be noted that magnetic transport can still become important for larger values of $\beta$. However, an increase in $\beta$ is beneficial for ITG suppression [20], which could lead to improved confinement regimes before triggering fast ion magnetic transport. Further analyses and experiments are then needed to clarify the phenomenology of this interaction.

\section{Conclusion}

The numerical platform recently developed to study the interaction between energetic ions and turbulence was presented in this work. The microturbulent fields resulting from the saturation of ITG/TEM instabilities were studied with the gyrokinetic code GENE. A dedicated set of velocity space-dependent transport quantities was introduced to obtain the fast ion diffusivity from the gyrokinetic simulation. VENUS, a guiding centre code, was then used to simulate the interplay between guiding centre drift motion, collisions and the stochastic diffusion induced by GENE's turbulence. Following previous investigations performed for ITER, which demonstrated that turbulence has negligible effects on fast ions, numerical simulations were carried out for DEMO and TCV. It was demonstrated that beam ions are redistributed in DEMO, where the large temperatures reduce $E_{\mathrm{nbi}} / T_{\mathrm{e}}$ and, more importantly, dilate the fast ion slowing down time. Some of the energy carried by the neutral beam ions is consequently given to peripheral plasma regions, thus undermining background ion heating and plasma performance. It was finally demonstrated that beam ion redistribution can be either strong or negligible in $\mathrm{TCV}$, depending on the plasma temperature achieved with the flexible ECH system. Therefore, the fast ion transport phenomenon could easily be investigated in the planned neutral beam heating upgrade of this flexible machine. Optimistic conclusions were drawn for alpha particles, whose large birth energy leads to strong gyroaveraging effects and no turbulent redistribution/losses. Negligible transport from magnetic fluctuations was also observed. 

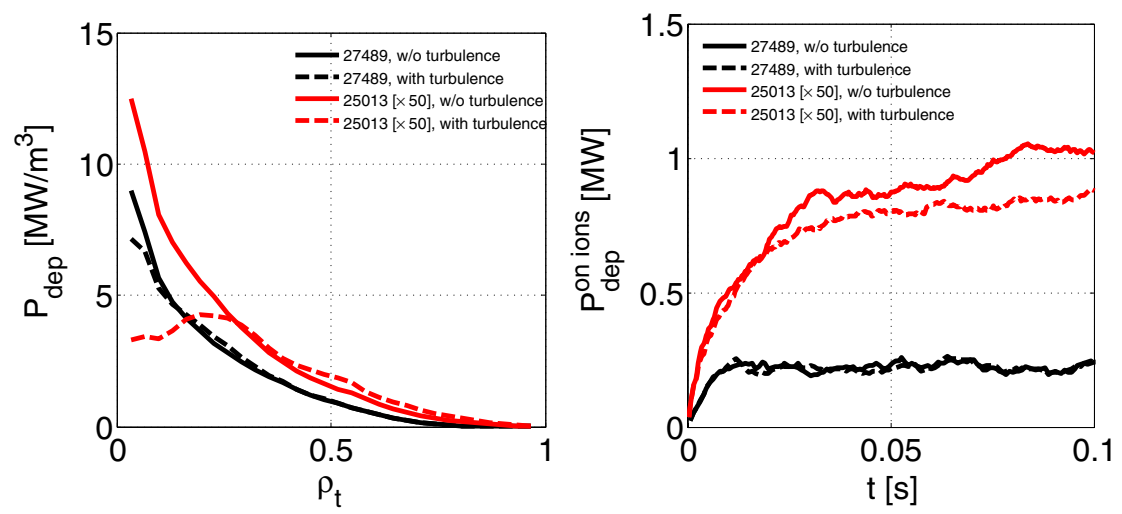

Figure 8. Fast ion heat deposition as a function of minor radius (left panel) and time (right panel) for plasma discharges \#27489 (black) and \#25013. Simulations are performed with and without the inclusion of microturbulent fields (dashed and sold line, respectively). The same colour and line code are used in the right panel, where the heat deposition on ions for the two discharges is shown.

\section{Acknowledgments}

The main author gratefully acknowledges insightful comments and suggestions by W. Heidbrink, F. Jenko and A. Karpushov. The authors would also like to thank the reviewers for their comments and suggestions to the first draft of this paper. This work was supported by EURATOM under the contract of Association with CRPP-EPFL, and was carried out within the framework of the European Fusion Development Agreement. This work was also partly supported by the Swiss National Science Foundation. The views and opinions expressed herein do not necessarily reflect those of the European Commission.

\section{(c) Euratom 2012.}

\section{References}

[1] Fasoli A. et al 2007 Progress in the ITER Physics Basis Chapter 5: Physics of energetic ions Nucl. Fusion 47 S264

[2] Heidbrink W.W. 2008 Phys. Plasmas 15055501

[3] Darrow D.S. et al 1997 Nucl. Fusion 37939

[4] Naitou H. et al 1979 J. Phys. Soc. Japan 46258

[5] Günter S. et al 2007 Nucl. Fusion 47920

[6] Heidbrink W. et al 2009 Phys. Rev. Lett. 103175001

[7] Hauff T. et al 2008 Phys. Plasmas 15112307

[8] Dannert T. et al 2008 Phys. Plasmas 15062508

[9] Zhang W. et al 2008 Phys. Rev. Lett. 101095001

[10] Albergante M. et al 2009 Phys. Plasmas 16112301

[11] Albergante M. et al 2011 Plasma Phys. Control. Fusion 53054002

[12] Hauff T. et al 2009 Phys. Rev. Lett. 102075004
[13] Fischer O. et al 2002 Nucl. Fusion 42817

[14] Isaev M.Y. et al 2006 Fusion Sci. Technol. 50440 www.new.ans.org/pubs/journals/fst/a_1267

[15] Graves J.P. et al 2012 Nature Commun. 3624

[16] Jenko F. et al 2000 Phys. Plasmas 71904

[17] Dannert T. et al 2005 Phys. Plasmas 12072309

[18] Ward D.J. 2010 Plasma Phys. Control. Fusion 52124033

[19] Fasoli A. et al 2009 Nucl. Fusion 49104005

[20] Pueschel M.J. et al 2008 Phys. Plasmas 15102310

[21] Heidbrink W. 2011 Irvine, private communication (University of California)

[22] Wesson J 2004 Tokamaks (New York: Oxford Science Publication)

[23] Albergante M. et al 2010 Nucl. Fusion 50084013

[24] Fable E. et al 2010 Plasma Phys. Control. Fusion 52015007

[25] Angioni C. et al 2009 Nucl. Fusion 49055013

[26] Testa D. et al 2012 Europhys. Lett. 9735003

[27] Lapillonne X. et al 2011 Plasma Phys. Control. Fusion 53054011

[28] Heidbrink W.W. et al 2007 Phys. Rev. Lett. 99245002

[29] Bass E.M. et al 2010 Phys. Plasmas 17112319

[30] Xu X.Q. et al 1991 Phys. Fluids B 3627

[31] Kuosela T. 2011 private communication (Aalto University)

[32] Brunner S. 2011 private communication (École Polytechnique Fédérale de Lausanne)

[33] Moseev D et al 2011 Plasma Phys. Control. Fusion 53105004

[34] Albergante M. 2011 Interaction between fast ions and microturbulence in thermonuclear devices $P h D$ Thesis Lausanne http://library.epfl.ch/en/theses/?nr=5164

[35] Gormezano C. et al 2007 Nucl. Fusion 47285

[36] Hein T. et al 2010 Phys. Plasmas 17012307

[37] Karpushov A.N. et al 2011 Fusion Eng. Des. 86868

[38] Marinoni A. et al 2009 Plasma Phys. Control. Fusion 51055016 